\title{
La intervención social en una «sociedad» reticular $^{1}$
}

\author{
Manuel Herrera Gómez \\ Universidad de Granada \\ mherrera@ugr.es
}

Carmen Alemán Bacho

Universidad Nacional de Educación a Distancia

caleman@poli.uned.es

\section{Resumen}

Uno de los retos del pasado, del presente y del futuro de los científicos sociales es la mejora de las formas de intervención en la realidad social. El modelo burocrático y asistencialista predominante desde los años cincuenta, muestra significativas carencias para hacer frente a las nuevas demandas de una sociedad cada vez más compleja y dirigida hacia la calidad de vida. En este trabajo se presenta una alternativa basada en la perspectiva relacional, desde la que los problemas sociales se interpretan a partir de una red problemática de relaciones, sobre las que se debe intervenir para buscar una solución adecuada. Esta orientación promueve una colaboración entre redes formales e informales que permite redefinir el welfare y que contribuye a un más atento y eficaz servicio de care a las personas.

Palabras clave: teoría relacional, intervenciones de redes, asistencia de comunidad, servicios de bienestar.

\section{Abstract}

One of the challenges of social scientists has always been, and will always be, the improvement of the ways of intervention in social reality. The bureaucratic and interventionist model, predominant since the 50's, shows significant lacks in order to meet the new demands of an ever increasingly complex society that is oriented towards quality of life. This paper presents an alternative model based on a relational perspective, from which social problems are interpreted in terms of a complex relational network. Solutions to social problems in this network of relations are reached by acting upon those relations. This perspective promotes a collaboration among formal and informal networks that allows us to redefine welfare and that contributes to a more efficient care of the persons involved.

Key words: relational theory, acting upon networks, community care, welfare services.

1. El presente trabajo forma parte del proyecto de investigación Las politicas migratorias de las Comunidades Autónomas españolas, financiado por el BBVA y dirigido por el Profesor Iglesias de Ussel, y es fruto de una estancia de investigación en el Center for Comparative Immigration Studies de la Universidad de California-San Diego en el curso académico 2006-2007. 


\section{Sumario}

1. Hacia una perspectiva relacional en el trabajo social

2. El trabajo de redes en las conexiones entre familia, marginación social y servicios de bienestar
3. ¿Qué «sistema de redes»? ¿Qué «asistencia de comunidad»? ¿Qué «intervenciones de redes»? Indicaciones metodológicas 4. Nuevos perfiles profesionales para un servicio social en red

Bibliografía

\section{Hacia una perspectiva relacional en el trabajo social}

De forma muy diferente a lo ocurrido décadas atrás, cuando la política social se centraba en la construcción de instituciones públicas (estatales) de bienestar, desde hace ya algún tiempo muchos hablan de "trabajo de redes», de «intervención de redes», de "servicios en red", de "sistemas en red», etc. (Folgheraiter, 1994; Di Nicola, 1998). Sin embargo, en el uso de estos términos, muy pocos van más allá de un ambiguo lenguaje cibernético y de ingeniería social. Otros, de forma más banal aún, se dejan engañar por el lenguaje sistémico-organizativo, como si las redes de las que se habla fueran composiciones de líneas y de nudos, con loops y feed-backs de diverso género, que se organizan según cualquier automatismo. Dicho en otros términos y de forma positiva, un «discurso de redes» necesita de una visión sociológica más apropiada, visión que, en la terminología que utilizaremos, se llama sociología relacional (Donati, 1986, 1991, 1993).

En estas páginas, nuestro objetivo es presentar las grandes líneas del planteamiento relacional, entendido como aquella perspectiva de lectura específica de la sociedad que está en la base del trabajo social de redes. Este planteamiento tiene como punto de partida dotarse de una representación y de un mapa del campo de análisis y de intervención: se trata de aquélla que denominamos «visión de la sociedad como realidad relacional». Enfoque que, desde un primer momento, conlleva un problema de doble orden: por un lado, saber crear y gestionar las relaciones sociales (actualizadas, potenciales y virtuales), de tal manera que se reduzcan las patologías y los efectos perversos, $y$, por otro, se mantengan las conexiones más significativas posibles entre las cualidades propiamente humanas de las relaciones sociales y los determinismos que incorporan. Posteriormente, se continúa con la focalización del "problema social», entendido como problemática de una red interactiva en la que los procesos de marginación, las condiciones familiares de los individuos y los servicios se confrontan entre sí. Observado de esta forma, el problema sugiere internamente instructivas modalidades de intervención que también son modos de re-pensar y re-activar la sociedad. De ahí, y en consecuencia, la necesidad de diseñar los nuevos perfiles metodológicos y profesionales de la intervención social. 
El trabajo social jamás es neutro con respecto a la sociedad. Las metodologías que plantea, y los contenidos de su profesionalidad, no son indiferentes respecto a la sociedad en que son aplicados: trazan las diferencias, «hacen las diferencias».

Trataremos de poner un ejemplo. El case work de los años cincuenta, de claras connotaciones anglosajonas, reflejaba una concepción individualista de la sociedad (y, en consecuencia, del trabajo social) que todavía está vigente, en el marco de una visión reparadora del Welfare State. La idea de la sociedad era la de un campo de condiciones y de individuos «ricos y pobres» como resultado de la suerte/no suerte individuales. Esta idea comportaba una concepción asistencialista de la ayuda social. Una concepción que se reforzaba, y no se atenuaba, por el marco meritocrático y profesional en el que se inscribe el trabajo social. En los años sesenta, y como respuesta a los movimientos de contestación social y juvenil, se ha ido afirmando en Europa, junto con las instancias liberadoras y emancipadoras, una idea "panóptica» de la sociedad, idea que se ha traducido en una teoría y en una práctica cada vez más sistémica del Welfare State y de sus intervenciones. Detrás de la idea de un Welfare State total, estaba presente una visión de la sociedad como organización funcionalmente estratificada. La sociedad asistida ha cambiado: ha sido leída e interpretada como un «sistema». Y, paralelamente, ha sido teorizado el nuevo trabajo social: un trabajo políticamente orientado y estructurado de tal forma para realizar una distribución funcional de los recursos y las oportunidades de vida en clave emancipadora, donde emancipación ha significado y significa "más inclusión» en las garantías del Estado social. En las décadas de los años setenta y ochenta, hemos contemplado la afirmación de este proyecto y, al mismo tiempo, hemos asistido a su relativo fracaso. ¿Por qué ha fracasado como proyecto global? Obviamente, las razones son muchas y diversas. Las podemos resumir en las siguientes afirmaciones: porque la sociedad industrial-burocrática se ha hecho "compleja» ${ }^{2}$. Por este motivo, nos podemos preguntar: en una sociedad de este género, ¿cómo puede entenderse y practicarse el trabajo social?

Reticularidad. Esta nueva característica, al mismo tiempo difusa y específica de nuestra sociedad, significa: difusión de «juegos relacionales»; afirmación de las identidades sociales mediante una especie de destrucción creativa de las solidaridades precedentes; creación y gestión de las características de "pertenencia» de las personas, no como construcción de relaciones sobre bases estables o adscriptivas, sino como continua negociación de relaciones elegidas mediante recorridos que no corresponden a lógicas funcionales o de la primera modernidad; crecimiento de formas asociativas, y también "grupales», que mezclan y combinan entre sí elementos informales y formales en el uso y con-

2. Sociedad compleja significa: orientada a generar diferencias, aún más, a contenerlas; más contingente en todos los actores y en todas las relaciones; capaz de dar vida a más posibilidades de cuantas puedan ser experimentadas y organizadas; una sociedad cada vez más incierta y arriesgada, pero propiamente como elección y como su intrínseca organización. En ella decaen las representaciones y las prácticas precedentes del trabajo social. 
sumo de las relaciones (Melucci, 1989; Laraña y Gusfield, 2001). Las metodologías y los nuevos perfiles profesionales del trabajo social deben trazar las debidas consecuencias.

\section{El trabajo de redes en las conexiones entre familia, marginación social y servicios de bienestar}

Buena parte del trabajo social tiene que ver con las conexiones entre situaciones familiares de los individuos, procesos de marginación y servicios públicos y privados de bienestar. ¿Por qué hablar de familia? Porque si deseamos desinstitucionalizar a las capas más débiles de la población (enfermos mentales, ancianos en residencias, discapacitados, infancia) por fuerza debemos pensar en la familia. Sabemos que el resto de comunidades (terapéuticas o de otro tipo) son sustitutas de la familia (Iglesias de Ussel y Meil, 2001).

Los conocimientos que el trabajador social debe de tener para desarrollar la acción de intervención aún tienen como objetivo, en general, saber en qué condiciones se encuentra la familia, cuáles son las necesidades en relación con su composición, estatus social y otras características relevantes, y cuáles son las respuestas que vienen dadas (o no) a tales necesidades, de qué manera, teniendo en cuenta la estructura familiar. Todo esto aún es necesario. Sin embargo, el trabajo de redes exige algo más y diferente. En una sociedad orientada a la "calidad de vida", ya no se puede aceptar una definición a priori (no relacional) de las necesidades y una configuración ya diseñada de los servicios. Se hace necesario definir y alcanzar un objetivo de respiro teórico y práctico más amplio: definir relacionalmente las necesidades y las formas de afrontarlas. El trabajo social, por tanto, debe redefinir el problema no como carencia de este o aquel factor, ya sea micro o macro, ni tampoco como una especie de proceso indefinido o indefinible. Debe comprender cómo la intersección entre interior y exterior de la familia, entre el mundo vital de las redes primarias y las instituciones de ayuda (representadas por los servicios públicos, privados y de tercer sector) define relacionalmente las necesidades de las familias, y si y cómo esta intersección busque relacionalmente ir a su encuentro.

El trabajo social no puede limitarse a contemplar si las dificultades se han debido a este o a aquel motivo o "causa» (por ejemplo: la ausencia de un trabajo, una enfermedad, la presencia de un anciano, de un discapacitado, etc.). No puede circunscribirse a observar si la familia "funciona» o "no funciona», ni en qué forma y medida lo hace o no. El trabajo social debe ir más allá de este planteamiento tradicional. El trabajo de redes trata de hacer esto redefiniendo el problema como una situación generada por una red problemática de relaciones. Y la solución debe buscarse en las acciones sobre relaciones, no en otro lugar.

El punto de partida es el siguiente: se plantea la hipótesis de que la familia es un sistema relacional que define sus necesidades «en relación» con el contexto y con sus sujetos. Y se asume que el sistema de atención es más o menos capaz de afrontar las necesidades familiares en la medida en que tiene en cuen- 
ta esta relacionalidad y se estructura así mismo «relacionalmente» en sus confrontaciones. Teniendo como telón de fondo esta simple hipótesis, a partir de la cual - teóricamente- se espera una correspondencia cualitativa entre organización familiar y organización de los servicios, el trabajador social puede comprender y medir el sentido y la funcionalidad de las acciones efectivamente activadas para responder a las exigencias de una vida cotidiana complicada, vivida con la pretensión de evitar la marginación de los sujetos más débiles. Todo esto nos traslada a la suposición de que un sistema de respuesta (coping) en las confrontaciones de las necesidades familiares, es más óptimo cuanto más asume la configuración de un «sistema complejo en red», en el que necesidades y respuestas están correlacionadas a través de una pluralidad de actores, formales e informales, de asistencia ${ }^{3}$.

El sentido y la utilidad de este marco de hipótesis es simple: se espera que, cuanto más existe el sistema en red y es más capaz de autoorganizarse, tanto más las necesidades pueden afrontarse de manera satisfactoria por los mismos sujetos que son portadores mediante oportunas ayudas. Con posterioridad, se observará si esto es cierto o no, por qué sí o por qué no, y con qué consecuencias. De las discrepancias entre la realidad esperada y la realidad de hecho se aguardan conocimientos instructivos. En el caso de que se verifiquen las hipótesis, se pueden extraer indicaciones útiles en el nivel operativo. En caso contrario, se deben reformular tanto las hipótesis como las indicaciones prácticas consiguientes. Por tanto, la primera asunción consiste en considerar la condición social de las personas, in primis familiar, como una red de relaciones en un sistema complejo que genera problemas así como puede generar soluciones. Ciertamente, y en función de cuanto se ha dicho, no se trata de ver a la familia como la «causa» de los problemas sociales. Más bien al contrario. Es más exacto decir que los problemas sociales emergen en la y a través de la familia.

Muchos trabajadores sociales piensan los «modelos» familiares como si fuesen "cosas», en el trabajo de redes, sin embargo, se trata de conocer y comprender las estructuras y los comportamientos de las familias no como modelos ya dados o ya estructurados, sino como sistemas relacionales variables en el tiempo que se modifican en relación con las exigencias de cuidado y atención de las personas más débiles. Esto es lo propio de los fines de la intervención profesional en el marco de una más amplia política social, coherente con la visión relacional. Si, en efecto, es verdad que las familias son redes relacionales complejas, sus necesidades son definidas como problemas de relacionamiento,

3. Obviamente, en el nivel empírico, se espera que tal sistema en red jamás sea plenamente satisfactorio, ni ya dado, ni perfectamente definido. Se espera, sin embargo, que estas redes sean, en la mayor parte de los casos, más desorganizadas que organizadas; con un alto compromiso y negociación, antes que consensuales y preordenadas; más suprafuncionales que funcionalmente bien específicas, $y$, en cuanto a los recursos y a los sujetos participantes, muy combinatorias e interactivas, más que unilaterales y normativizadas. La red puede ser más o menos consciente, más o menos eficiente, más o menos eficaz, más o menos óptima en función de una serie de variables que intervienen y de las que debe dar cuenta el trabajador social. 
y así también para las soluciones de tales necesidades. El relacionamiento es muy interesante si se contempla desde el punto de vista de quien se dedica más intensamente al cuidado y a la atención de las personas débiles (llamado care giver), que, en la mayor parte de las ocasiones, es la mujer (esposa, madre, hija), en cuanto que el care giver es un poco la "estela sociográfica» en el centro de la red de apoyo, y por tanto es la persona más participante en ella. Es el care giver quien debe sostener la funcionalidad de la red. Por otra parte, se trata de ver cómo este sistema complejo de relacionamientos se organiza según la clase (o estatus) social de la familia y del contexto territorial ${ }^{4}$.

El planteamiento de redes nos ubica ante una realidad muy lejana de las descripciones asépticas, puramente estadísticas —especialmente demográficas- que siempre nos describen a la familia como cada vez más aislada y fragmentada, como si la solidaridad se estuviese agotando ${ }^{5}$. Aunque es cierto que en Europa aumentan los singles y las parejas sin hijos, y que muchas investigaciones describen familias cada vez más «despedazadas» a continuación de la división y del conflicto lacerante, no por ello es lícito deducir que la familia progresivamente se reduce, o que abandone o aísle a sus miembros más débiles (Iglesias de Ussel, 1998). Es en las situaciones más desesperadas, sin embargo, donde la observación empírica revela y experimenta la familia como mediación social, y la fuerza persistente de esta mediación, que es más fuerte cuanto más en riesgo de marginación total se encuentra una persona débil (Pérez Díaz, 1998).

Incluso entre los jóvenes problemáticos o no adaptados esto es cierto. Es muy raro encontrar menores para los que la familia no existe tout court: posiblemente existen «trozos» de familia, relaciones mutiladas, truncadas, violentas, pero no existe el vacío, sino como resultado de un proceso que - también en los momentos de mayores dificultades - tiende a mantener un mínimo de red. Esto también es cierto en los casos de pobreza ${ }^{6}$. De hecho, donde existe

4. Es oportuno señalar que, en este planteamiento relacional, se adopta una concepción dinámica, pero no relativista de la familia. Ésta última, la familia, cambia en el tiempo y en el espacio, pero no es algo que podamos pensar como queramos. Tiene sus reglas. Puede ampliarse (por ejemplo: con el ingreso de un miembro) o reducirse (por ejemplo: con la salida de un miembro), y ciertamente cambia las propias necesidades según el ciclo de vida y de sus transiciones. Pero lo que la constituye como familia, es decir, su íntima relacionalidad como reciprocidad "tendencialmente plena» — al mismo tiempo de mundo vital y sistémica- entre géneros y generaciones, no se pone en duda. De hecho, no todos los trabajadores sociales profesionales ven que la familia «existe», en el sentido de que ésta — normalmente- no «escapa» ante acontecimientos difíciles, como la pérdida de recursos, el nacimiento de un hijo enfermo, la presencia de un discapacitado adulto, la pérdida de un progenitor (a continuación de la separación, el divorcio o la muerte) o la llegada de la ancianidad. Al contrario, acontecimientos como éstos, en la mayor parte de las ocasiones, dan un nuevo impulso a la movilización de fuertes solidaridades latentes que, sin embargo, sólo consiguen organizarse si existen recursos de redes, de otra forma se dispersan.

5. Un buen ejemplo son las Statistiques démographiques elaboradas por Euroestat.

6. Hay quienes, tras unos cuantos años de investigación de campo, actualmente llegan a la conclusión de que los "pobres no existen», que ya no los debemos llamar "pobres», sino 
necesidad, la familia consigue activar sus fuerzas solidarias. Las razones por las que puede hacerlo son muchas y diversas según los casos. No entraremos aquí en estos aspectos. Ni tampoco entraremos en el tema sobre cuánta solidaridad es positiva o negativa a los efectos relacionales, es decir, para los efectos que tiene sobre la dinámica de las relaciones y sobre singulares personas en la fami$\mathrm{lia}^{7}$. En cualquier caso, debemos constatar que la familia no escapa de las propias responsabilidades. Cuando lo hace es porque, en la mayor parte de las ocasiones, no hay alternativas. Es decir, cuando no tiene una adecuada red de apoyo que ayude a quien quiere o debe ayudar a los más débiles.

¿Cómo puede una familia hacer frente a condiciones de vida cada vez más inestables o insoportables, como, por ejemplo, cuando un solo progenitor debe trabajar o un anciano debe sostener a un progenitor más anciano que él o ella, cuando hay un hijo adulto totalmente inválido quizás en presencia de un padre desocupado o de una madre enferma? La respuesta es que la familia no podría hacerlo si no hubiese una red "entorno" y sobre todo una red primaria constituida por parientes, vecinos, amigos y voluntarios «espontáneos» (a título personal) (Requena, 1994, p. 73-90). Disponer o no de tal red «informal» es crucial para la capacidad de sostener las solidaridades familiares y oponerse a los procesos de marginación de los miembros débiles ${ }^{8}$.

¿En qué medida el servicio social tiene en cuenta todo esto? Estamos ante un punto fundamental en el que es necesario centrar la atención. A pesar de su enorme relevancia, la red informal generalmente no es reconocida por los

«residuos» (Ayala Cañón y Martínez López, 1999). Detrás de esta apreciación, se esconde una cierta idea del servicio social en el marco de una ciudadanía entendida como derecho a ser mantenidos sin ninguna responsabilidad por parte de quien recibe la ayuda social (ideología del puro entitlement). Antes que una conclusión aberrante desde el punto de vista humano, la idea de que "no existan personas pobres, sino solamente residuos sociales», es un error sociológico: no se contemplan las redes que generan estas situaciones. Condiciones y personas «residuales» son el producto de un déficit en la visión de la sociedad como realidad relacional: no se observa cómo — de hecho — las relaciones son destruidas, manipuladas, colonizadas. La idea de servicio social que sostiene tal visión no puede ser otra que la asistencialista, caritativa, burocrática, antes que inspirada en los derechos humanos, auténticamente solidaria y responsable (Di Nicola, 1998, p. 150-195).

7. Véanse Wilmott (1986), Sanicola (1990), Laville (1992), Folgheraiter y Donati (1993), Herrera (1998), Requena (1999), Iglesias de Ussel y Meil (2001) y Hernández Rodríguez (2001).

8. Una de las más recientes investigaciones empíricas sobre el contexto italiano ha verificado que la mayoría de las familias tienen una importante red informal, en el que un porcentaje medio contempla al menos dos tercios del total de las familias, y puede ser mayor o menor según el contexto territorial, mientras que, en el tercio restante de las familias, la red puede ser más o menos significativa, pero nunca totalmente ausente. En todos los casos, la red informal modifica el sentido práctico de la vida familiar, sus necesidades, sus intentos de respuesta a las necesidades (Prandini, 2006, p. 68-108). Por tanto, resulta esencial para poder afrontar las necesidades, incluso las más pequeñas de la vida cotidiana. Sin esas redes, las familias no podrían encontrar aquel mínimo de recursos para hacer viables las situaciones más estresantes y de mayor dificultad. Naturalmente, esta red varía en el tiempo y en el espacio según una amplia gama de factores de los que no nos podemos ocupar. 
denominados servicios «formales» (servicios públicos del Estado) ${ }^{9}$, siendo, en buena parte de las ocasiones, una alternativa a estos últimos: si existen las redes informales, los servicios sociales actúan en otro lugar, van allí donde la red falta o piensan que falta. En consecuencia, no se activa ninguna colaboración, y los dos mundos (familias y servicios públicos) quedan separados, con graves disfunciones, costes más elevados y la frustrada sinergia de motivaciones y competencias entre los sectores privado y público $^{10}$.

¿Cuáles son las causas de este estado de la situación? La ausencia de colaboración entre redes formales (profesionales) e informales (de la parentela a los grupos de amigos y de voluntariado más espontáneo) puede reconducirse, por una parte, hacia las familias, que en alguna ocasión tienden a encerrarse en sí mismas y recurren a los servicios públicos sólo cuando la red informal está ausente o es muy carente, y, por otra, a la forma de actuar de los servicios institucionales que no "ven» a las redes sociales ${ }^{11}$.

En cuanto que corresponde a los servicios públicos formales el objetivo profesional de activar relaciones más comunicativas y colaboradoras con las familias, incumbe a estas últimas la responsabilidad — no indiferente- de tomar conciencia de este estado de la situación y de modificarlo. Se impone un nuevo estilo de formación de los operadores y un nuevo diseño del sistema de servicios para el bienestar. ¿Cuál? Dando una respuesta muy breve, podremos decir: ubicando a la familia en el «centro» de la comunidad local y organizando los servicios como redes formales e informales de apoyo a sus funciones. Se trata de ver cómo se puede hacer esto.

En la actualidad, crece el número de aquéllos que reconocen que es necesario reforzar a la familia si queremos tener un mundo más humano para hacer

9. Véanse Bulmer (1989), Herrera (1998), Flaquer (2004) y Prandini (2006).

10. El mismo tercer sector aparece seducido por esta lógica. Especialmente el voluntariado más organizado con frecuencia está ausente cuando la familia trata de resolver los problemas por sí sola, incluso con grandes sacrificios y tensiones, mientras que está presente cuando debería intervenir el servicio público, al cual suple (Herrera, Castón, 2003). El voluntariado tiene necesidad de repensar completamente sus subjetividades y su capacidad técnica de intervención. Diferente es el voluntariado local y menos formalizado, que se presenta más flexible y capaz de relacionarse en las confrontaciones de las necesidades de la familia, aunque indudablemente es necesario promover una "cultura de la acción voluntaria» del singular y de los pequeños grupos, dotándola de mayores instrumentos, habilidades y reflexividad (Rossi, 1999, p. 5-25). Muy baja es la "propensión teórica» a recurrir a servicios de mercado (es decir, de pago). Pero, de hecho - especialmente ciertas necesidades, como las referentes a ancianos-, soy muy utilizadas en los contextos en que existe un mercado privado que las ofrece (Castón, Ramos y García, 2004; Trinidad, 2005). Casi ausente (especialmente en los países mediterráneos) es la valoración de las posibles sinergias entre el sector privado de mercado y el privado social, debido a una fuerte mediación ejercida por el Estado sobre el privado social (Salinas Ramos, 2001).

11. Algunas investigaciones han verificado que ambas causas desempeñan un cierto rol (en medida diferente según las situaciones). Al mismo tiempo, se puede demostrar que estas dos causas están correlacionadas entre sí (Carrà, 2002, p. 213-256). Dicho en otros términos, los comportamientos de las familias son especulados (y vienen reforzados) por análogos comportamientos por parte de los operadores públicos. 
frente a los dramáticos problemas del Estado de bienestar ${ }^{12}$. Aún más, se es consciente de que muchos de los problemas sociales nacen del siguiente hecho: la familia no está en situación de asumir sus objetivos. Quien comparte esta idea no es una minoría, como ocurría hace algunos años. En los comienzos de nuevo milenio, la familia es revalorada. Ahora bien, es necesario entender cuál es el sentido de esta revalorización.

Dos problemas relevantes están en la base del planteamiento relacional: a) Incluso cuando se es consciente de la "centralidad» de la familia, ¿a qué «forma de familia» debemos aludir? Las respuestas son inciertas y ambiguas, porque no se consiguen identificar los caracteres y trazar los límites de la familia. El planteamiento relacional no da una respuesta a la carta. Indica que la familia debe ser analizada como "red solidaria de apoyo", es decir, como relación de reciprocidad entre géneros y generaciones, en constante conexión con el exterior. No es raro que, cuando muchos plantean el problema de la «forma familiar", emerja una realidad desconcertante: el Estado social penaliza la agregación familiar, y la penaliza cuanto más solidaria es. Desde este perfil, resulta que las familias no sólo se hacen cargo de los propios miembros débiles, sino que lo hacen debiendo superar todas las barreras y penalizaciones que contra esta solidaridad plantea la colectividad políticamente organizada; b) Incluso cuando estamos de acuerdo en la necesidad de ser conscientes de que los sistemas de Welfare State han encontrado ciertos límites, ¿quién debe ocupar su lugar y quién asumirá los objetivos? ¿Sólo la familia? Evidentemente, no, la cosa sería imposible, ello significaría la vuelta a la beneficencia y a la caridad del pasado. En la actualidad, la respuesta es incierta: muchos piensan que las funciones de gestión que hace un tiempo eran asumidas por el Estado, deben ser recogidas ahora por el mercado (De Leonardis, 1996), en cambio, otros consideran que puede y debe asumirlas el privado social (en primer lugar: voluntariado, asociacionismo social, cooperación de solidaridad social) (Hirst, 1997). También sobre este punto la observación relacional es ilustrativa. Aclara que no es tanto un singular actor el que cuenta, sino sus relaciones. Evidentemente, debe haber una pluralidad de actores. Pero la pregunta más crucial es: ¿cómo juegan y cómo deberían jugar todos estos sujetos? La respuesta que puede obtenerse de la observación empírica es la siguiente: estos actores aún no han aprendido a jugar cooperativamente entre ellos. Ahora es cuando comienzan a observarse (y a reconocerse) recíprocamente, pero estos reconocimientos dan la sensación de generar conflictos y no formas cooperadoras y sinérgicas de acción. ¿Qué podría conducirlos a madurar reglas de un mejor juego? La respuesta está aquí: un sistema de redes.

12. Véanse, al respecto, las numerosas investigaciones, recomendaciones e informes elaborados tanto por los diversos organismos internacionales, como en todos los países europeos —entre otros, recordemos el Rapport Gilliand (1988), el Rapport Pini (1988), el Rapport Linke (1989) y el Rapport Laroque (1989); en España, puede verse Fundación Foessa (1995), Iglesias de Ussel (1998), Flaquer (1998, 2000), Alberdi (1999), Meil (1999), Muñoz, García Delgado y González Seara (1999), Del Campo (2003) y López López (2006). 
Más adelante, veremos qué significa esta expresión. Por el momento, nos gustaría subrayar que las intervenciones de política social para la familia actualmente deben repensarse a la luz de que aún son demasiado rígidas: presuponen un modelo de familia estandarizado y fijo en el tiempo que es totalmente irreal, especialmente en el caso de las situaciones de mayor debilidad social. En otros términos, las políticas sociales deben hacerse más flexibles respecto a las exigencias de las familias como sistemas relacionales que se modifican en el espacio y en el tiempo, en función tanto del tipo de personal débil a asistir, como del ciclo de vida de la misma familia. Desde una perspectiva de amplios horizontes, se trata de construir un sistema de protección social basado en «sistemas relacionales» que tengan en cuenta las modulaciones espacio-temporales de las necesidades familiares, mediante nuevas combinaciones de intervenciones formales e informales, públicas y privadas. La expresión clave es aquí asistencia de comunidad (community care), es decir, una asunción de la comunidad y por parte de la misma comunidad, que considere a la familia como sujeto y no sólo como destinatario pasivo de los servicios, en concreto sociosanitarios. Si la familia está insertada en un sistema relacional, es sobre este sistema sobre el que debe hacerse el diagnóstico de los problemas y al que va dirigida la terapia social. Si la asistencia es un sistema de redes, es necesario que las intervenciones para el bienestar sean «intervenciones de redes».

\section{3. ¿Qué «sistema de redes»? ¿Qué «asistencia de comunidad»? ¿Qué «intervenciones de redes»? Indicaciones metodológicas}

Trataremos ahora de diseñar, brevemente, qué nuevas iniciativas pueden tomarse, su framework general, las premisas de las que nacen, las condiciones que requieren para ser o hacerse eficaces y útiles, las dificultades que deben superarse, así como la creatividad que debe ser activada.

Antes de entrar de lleno en este tema, nos gustaría recordar que esta nueva forma de observar los problemas sociosanitarios ya tiene su historia, que parte de la declaración de la OMS de Alma Ata (1978) y llega a varias resoluciones del Parlamento europeo. El contenido de esta línea está en considerar posible que a los miembros débiles de la sociedad, desde los niños hasta los ancianos, es necesario no sólo consentir, sino afrontar los problemas sociosanitarios en su ambiente de vida, haciendo que todo el sistema de asistencia se configure como una amplia red de apoyo; se considera que los familiares que asumen el cuidado del niño, del discapacitado, del enfermo, del anciano, deben poder contar con un sistema de ayudas formales e informales que tenga las características de una comunidad de apoyo, de tal forma que el objetivo no recaiga exclusivamente en las mujeres. Con estas directivas en mente, trataremos de entender por qué y cómo tal política social es inevitable, mejor aún, deseable, al fin y al cabo, más humana para todos los sujetos participantes, no sólo para los enfermos en sentido estricto, sino también para todas las personas socialmente débiles, para los mismos care givers, es decir, para aquéllos que asumen seriamente sus problemas. 
La condición de las personas socialmente débiles tiende a mutar profundamente, si se deja a sí misma, sobre todo en lo referente al tejido de las relaciones sociales que estas personas tienen con su mundo cotidiano. Se han revelado algunas líneas de tendencia fuertes: por una parte, el creciente aislamiento físico-social de las personas clasificadas socialmente marginadas y, por otra, su institucionalización en lugares ad hoc, que tienden a segregarlas (Fargion, 1997). La tendencia al aislamiento de las personas débiles sólo en parte es resultado de las modificaciones de las estructuras familiares. A estos aspectos hay que añadir, por un lado, la difusión de planteamientos culturales que enfatizan el individualismo de los estilos de vida y, por otro, la presencia de respuestas rígidas por parte de los servicios sociales, sanitarios, educativos e incluso de tiempo libre, que encuentran más cómodo o más económico gestionar a la persona problemática (anciano, discapacitado, niño carente de asistencia) individualmente, sin tener en cuenta y sin englobar a la familia (a la que se apela cuando el sistema formal de los servicios no consigue afrontar todo aquello que se debería y necesita «recurrir» a la familia).

Contra estas tendencias macroestructurales hacia un creciente aislamiento social (hasta el punto de que algunos ya comienzan a invocar "políticas del ligamen social») (Carrà, 2002; Pandini, 2006), durante la última década, las investigaciones sociológicas han revelado que la salud psico-físico-social está positivamente correlacionada con la densidad, el significado y la funcionalidad de las redes informales en que viven las personas (Dennis, Catherine, 2003). El aislamiento es relativo, en el sentido de que subsisten, aún más aumentan, las redes informales que, si no en acto, al menos potencialmente, pueden englobarse (no tanto y no sólo a la parentela, sino también a los grupos de amigos, de vecindad, de voluntariado, de self-help y ayuda mutua). Estas redes sólo en parte son retazos tradicionales (especialmente presentes como tales en las áreas rurales y más periféricas), mientras que en buena medida - y siempre de forma más significativa- (por cualidad más que por cantidad) son emergencias sociales, es decir, relaciones creadas por los más recientes procesos de modernización en contextos urbanos. En ambos casos, viejas y nuevas redes informales no son reconocidas desde el punto de vista social, económico y político. Para ellas, aún no existe una representación social adecuada, que las valore y las inserte en un mundo vital significativo y funcional o en un servicio sociosanitario formal bien organizado.

Ante esta situación, se ha difundido la idea, y también una cierta práctica, de asistencia a domicilio o familiarizada (home care). El concepto cubre un amplio espectro de intervenciones aún no bien identificadas en sus potencialidades y requisitos específicos. Va desde la asistencia a domicilio, hasta la hospitalización a domicilio, hasta otras formas de asistencia en la habitación del anciano, del discapacitado, del enfermo, del niño «carente» de asistencia. En alguna ocasión, la asistencia domiciliaria va acompañada de "visitas» de informal helpers y, en los contextos más modernizados, de apoyos de telefonía social. La práctica, sin embargo, está bajo expectativas. Indudablemente, se trata de intervenciones útiles para una mejor asistencia. Ahora bien, la home care, sólo 
concebida de forma funcional, fácilmente termina por identificarse con «más asistencia» técnica, que no resuelve la soledad existencial y propiamente social, es decir, relacional, de la persona débil, aunque más bien deberíamos decir de la «relación débil». Si, desde el punto de vista psicofísico, débiles son las personas, desde el punto de vista sociológico es cierto que la debilidad es sobre todo de las relaciones.

Se deben activar redes de contacto, apoyo psicológico, intercambios de vida cotidiana, para permitir a la persona débil, según su grado de autosuficiencia, el máximo de autonomía vital. En otros términos, la vida de quien está —al menos potencialmente - en riesgo de marginación, va lo más «normalizada» posible, en el sentido de que se le deben dar oportunidades de vida normal relativamente a la cualidad de su condición. Sólo puede realizarse si el conjunto de los servicios formales e informales es concebido como community care y si esta última es pensada y practicada como línea o criterio directivo de política social (en el sentido de policy), y no como resultado espontáneo, sino como objeto de precisas políticas sociales, sanitarias, formativas y de apoyo.

Afloran una serie de implicaciones operativas para los servicios sociosanitarios, especialmente, la necesaria coordinación entre aspectos sociales y sanitarios, que, en los últimos años, no ha avanzado mucho. Y, posteriormente, una presencia significativa y no residual de lo informal, oportunamente sostenida por el ente público, en general el ente local, que debe adoptar un planteamiento y una práctica no instrumental hacia él. Pensemos en las nuevas formas de intervención, como el llamado "hospital a domicilio», la creación de una red ad hoc mediante un grupo de vecinos o de ayuda mutua, o un club informal, con la presencia de un operador profesional o semiprofesional de apoyo.

¿Qué puede aflorar para los niveles sociales y sanitarios, pero también formativos y especiales, en el plano regional y local? ¿Cómo deben ser reestructuradas las intervenciones formales? ¿Qué competencias deben demandarse al personal profesional e informal? ¿Cómo promover, y no sólo reconocer, la idea y la práctica de la community care en cuanto a política del bienestar globalmente entendido? La propuesta, aún emergente en Europa, es que se debe repensar completamente la política sociosanitaria como política de la comunidad local por y de parte de la misma comunidad observada desde la óptica de las redes familiares (Payne, 1999). Esto comporta una profunda reorientación de las políticas de los servicios, no sólo sociales y sanitarios, centrales y locales, y de los «planes» relativos. La programación debe cambiar de filosofía, en el sentido de que el Estado (central, autonómico y local) y los actores institucionales deberían orientarse a emancipar una caring society, actuando de «ordenadores generales» y no como "gestores totales» ${ }^{13}$.

13. Dicho en otros términos, correspondería a los servicios públicos y a sus operadores crear aquel puente entre asistencia formal y asistencia informal a través de: un trabajo de rating social; una lectura correcta de la demanda asistencial; una valoración de la demanda asistencial usando el filtro de las redes sociales, en concreto las redes de apoyo; una 
El rol de las nuevas iniciativas de privado social (formas de cooperación de solidaridad social, voluntariado incluso semiprofesional, mutualidades, asociaciones de ayuda y de ayuda mutua, formación de agregaciones autogestionadas, etc.) no pueden pensarse como residuales o puramente integradoras allí donde emerge la necesidad ex post, sino como elección estratégica preventiva de la necesidad, como estructura permanente, si se entiende diseñada dentro del marco de un Estado social que no abdica de las propias responsabilidades colectivas $^{14}$.

La posible solución de los problemas de las personas marginadas, en situaciones de desventaja, aisladas, en riesgo, y en general de quien tiene necesidad de asistencia y cuidado orientado a la persona y dimensionada sobre su situación familiar, cada vez más requiere una visión menos dependiente del Estado, menos burocrática y «activada» por aquélla tradicional puesta en acto por los operadores formales, lo que otorga un nuevo rol a la sociedad como sociedad "civil». Esta forma de acercarse a los problemas sociales supone interpretar el welfare de una forma activa, es decir, el paso desde unas políticas sociales asistencialistas o residuales a otras pluralistas y participativas, en las que se tenga en cuenta la potencialidad y capacidad de todos los actores que intervienen en la producción de bienestar social.

Los expertos saben que las políticas públicas, y los servicios sociales relativos administrados por operadores dependientes de los entes públicos, hasta ahora han sido pensados de forma inadecuada, y se apoyan sobre una serie de asunciones que tienen bases sociológicas muy frágiles, concretamente porque no tienen en cuenta que la ayuda sociosanitaria no es sólo un objetivo "formal» de organizaciones emanadas directamente del Welfare State, sino que también es perseguido y realizado por «actores informales». El haber olvidado y descuidado a las redes informales, ha llevado a resultados pobres y a fracasos. El resultado ha sido y es que, en el núcleo de la política pública de social care, existe un vacío que, con toda probabilidad, lleva a provisiones ineficaces o a deterioros para las personas en condición de necesidad. Si se quiere intervenir de forma correcta, por tanto, se debe pensar y organizar sistemáticamente la intervención como colaboración entre redes formales y redes informales.

Las redes informales no son la "solución total», pero son indispensables. Y los operadores sociosanitarios no pueden eximirse del deber de aprender a cómo construir sistemas de inclusión y distancia con ellas. No se trata de hacerse ilusiones. No debemos crear mitos. La redes informales son débiles y necesitan, a su vez, ser sostenidas. Las direcciones del cambio social llevan cada vez

descomposición de la necesidad en todas sus dimensiones; el establecimiento de "paquetes» asistenciales flexibles y diferenciados, construidos a partir de todos los recursos posibles que se deben y se pueden activar.

14. Recuerdo que este "tercer polo asociacional» se presenta hoy como el más dinámico, activo y capaz de absorber la ausencia de regulación que existe en el mercado, de igual forma que evita la alineación de una sociedad burocrática por vía estatal. Sobre el concepto «tercer polo asociacional», véase Hirst, 1998, 1999; Hirst y Bader (eds.), 2001. 
más, especialmente en las grandes áreas metropolitanas, a redes primarias (familiares y de parentesco) más dispersas, fragmentadas, con menores oportunidades de tener informal carers (parientes, familiares, amigos, vecinos, voluntarios espontáneos) a disposición. Al mismo tiempo, se está activando un proceso posterior de privatización de la sociedad, aumenta el número de las personas solas, aisladas, que no pueden disponer de ayudas informales (Iglesias de Ussel y otros, 2001). Estos fenómenos indudablemente crean perplejidades y dudas respecto al hecho de que la comunidad pueda dar servicios en el lugar del Estado. Pero, si se estudia bien el problema, se puede observar que sólo creando contextos de comunidad se podrán afrontar aquellas patologías sociales crecientes que las sociedades complejas, intrínsecamente arriesgadas, llevan consigo.

Se trata de encontrar la clave justa para no minusvalorar ni valorar en demasía la importancia y el rol de la community care como asunción de la comunidad por parte de la comunidad, en todos sus componentes, profesionales y no profesionales. Como cualquier otro planteamiento o instrumento de intervención, también puede utilizarse de forma distorsionada y ser instrumentalizado. Ciertamente, los objetivos son ambiciosos, pero la sociedad del naciente tercer milenio no puede renunciar a ellos, bajo pena de una alineación y una emancipación cada vez más difundida. Es necesario señalar que la implementación de las líneas de la community care aquí planteadas significa un crecimiento de las capacidades de todos los operadores, en primer lugar de los formales, pero también de los informales, para que: a las personas necesitadas de ayuda les sean dados los servicios más correctos en relación con su situación familiar, y en tiempos adecuados a sus particulares necesidades; y las personas sean ayudadas a vivir en el seno de sus familias el periodo más extenso posible, o bien en un ambiente lo más parecido al doméstico, de tal forma que las residencias y las asistencias institucionalizadas se reserven a aquéllos para quienes la necesidad no puede atenderse de otra forma.

Éstas son las finalidades de los servicios de redes y de la intervención de redes. Muchas propuestas se concentran principalmente sobre los medios. Esto se debe a que el nudo está propiamente en la ausencia de una «cultura de los instrumentos relacionales» que conlleve un más atento y eficaz servicio de care de las personas. Es oportuno señalar la importancia de que toda comunidad local asegure, tanto a nivel técnico como político, que exista alguien encargado de gestionar las políticas de community care que disponga del control sobre los recursos necesarios. Sólo de esta forma se podrá ayudar a las familias débiles o en riesgo de marginación; puesto que se crearán nuevas oportunidades para mejorar la calidad de los servicios disponibles y se obtendrán mejores prestaciones en relación con los costes. Lo que hasta el día de hoy ha sido un progreso "fuera de la norma», debería convertirse en norma.

Tratar de remendar el sistema tradicional de welfare, centralizado y estandarizado, no permitiría contemplar el problema nuclear de la creación de nuevas redes y desperdiciaría los beneficios que pueden obtenerse con una acción más concentrada en casos y las situaciones valoradas de forma específica. Existe 
la oportunidad de crear una partnership en la oferta de los servicios —entre el gobierno central, autonómico y el local, entre los servicios sociales y los sanitarios, entre las administraciones públicas y los sectores privados y voluntarios, entre los profesionales y los individuos-, lo que beneficiaría a aquéllos que están en situación de necesidad. El rol de sector público es esencial para asegurar que la asistencia efectivamente se realice, mientras que el cómo son dadas, es una consideración importante pero secundaria. Las autoridades locales deberían mostrar que están en situación de obtener y de dar valor real, no oportunidades abstractas.

\section{Nuevos perfiles profesionales para un servicio social en red}

En relación con los planteamientos y las metodologías planteados, esbozamos ahora las indicaciones referentes a los nuevos perfiles profesionales para los operadores sociales. Por desgracia, aún son muchas (demasiadas) las escuelas de trabajo social, por no hablar de los que preparan a otros profesionales (sanitarios, administrativos, jurídicos, etc.), que están ligadas a los viejos modelos profesionales típicos de la configuración tradicional (asistencial-burocrática) del Welfare State. Olvidan que, con la crisis del Estado de bienestar, su desestructuración y pluralización, la posición estructural de los operadores sociales se modifica radicalmente ${ }^{15}$. Sólo teniendo como telón de fondo el escenario anteriormente planteado se puede comprender el espíritu y los contenidos de las propuestas para nuevos modelos formativos y activos del operador social. Intentaremos trabajar las principales características: finalidad del servicio, dimensiones principales del perfil profesional, objetivos de competencias y responsabilidad.

Las principales finalidades del servicio en que los nuevos perfiles profesionales se insertan, deberían: a) ubicar a la persona débil en situación de vivir una vida lo más posiblemente normal en su ambiente, generalmente de tipo familiar, en la comunidad local; b) elaborar el paquete de servicios y apoyos necesarios para ayudar a la persona débil a alcanzar y a mantener el máximo posible de autonomía; se trata de activar intervenciones que operen de tal forma que puedan sostener todas las habilidades de la persona dependiente $y$ su pleno potencial de vida; c) dar a las personas más voz respecto a la forma en que viven su condición desventajada, y respecto a los servicios de los que tienen necesidad para ser ayudados a vivir lo más plenamente posible su vida;

15. El sistema de bienestar ya no tiene un «vértice» (un poder superior de referencia para todos); el sistema de bienestar ya no posee un "centro", ni de coordinación ni de dirección; el sistema de bienestar ya no dispone sólo de una o dominante imputación de responsabilidad colectiva; el sistema de bienestar se expande uniformemente en todas las direcciones; el resultado es que cualquier punto se distancia de los otros, pero también se encuentra ligado al movimiento general que afecta a todos los puntos en los que el bienestar se produce y consume; el bienestar se hace "autopoiético», es decir, debe producirse en el interior de cualquier sistema organizativo según su propia distinción y directriz. 
d) promover el desarrollo de una red de servicios entre el sector estatal, el sector privado social y el privado mercantil, que permita respetar estándares de buena calidad y adecuada profesionalidad; e) las autoridades públicas no deberían utilizar el privado social y el mercantil como instrumento de intervención residual o como agencia donde descargar las responsabilidades colectivas, sino que deberían actuar como apoyo de agencias autónomas del privado social y como reguladoras del privado mercantil, de tal forma que se acreciente el radio de las posibilidades y de las elecciones de los usuarios; f) aclarar las responsabilidades recíprocas de las agencias formales y del privado social, de tal forma que se haga más fácil y transparente su proceso de rendir cuentas, y g) introducir una nueva estructura de financiación para las atenciones sociales que asegure un valor más elevado de las prestaciones dadas a cambio de dinero fiscal.

Las principales dimensiones o componentes de los nuevos perfiles profesionales propios del operador social deberían ser tales que permitieran desarrollar las siguientes capacidades: a) responder de forma flexible y sensible a las necesidades de las personas, de sus familias y de quien los asiste, creando redes apropiadas entre sí; b) permitir un amplio espectro de opciones de redes a los usuarios, en concreto, mediante la utilización de medios diversificados (en especie o en dinero, y también éstos diversificados por tipos) y flexibles (con posibilidades de cambiar fácilmente en el desarrollo); c) no intervenir más de lo que sea necesario, de manera que sea posible acrecentar la autonomía del usuario y/o de su familia en el contexto de los grupos sociales de pertenencia, es decir, como capacidad autónoma de «hacer red», y d) practicar una ayuda activa de las personas que asisten a otras, los informales carers de ancianos, discapacitados, niños dependientes, etc., con medidas y programas ad hoc que tengan estructura relacional.

Los objetivos específicos de competencia de los nuevos perfiles profesionales para los operadores sociales deberían orientarse hacia las finalidades siguientes: a) promover las capacidades de desarrollar servicios domiciliarios, servicios diurnos de apoyo (como aquéllos denominados «de respiro») que permitan a las personas vivir en sus familias siempre que sea factible y sensato; es necesario darse cuenta de que las formas de organizar los servicios y los sistemas de financiación de las intervenciones, hasta ahora han operado contra esta línea; b) asegurar que quien tiene el objetivo de elaborar servicios sociosanitarios dé prioridad a un apoyo efectivo del personal de cuidado, y c) elevar la calidad de las intervenciones mediante una apropiada evaluación (assessment) de las necesidades y una mejor competencia para la "gestión del caso» (case management) (los paquetes de asistencia deben elegirse en relación con las situaciones específicas y lo más posible de acuerdo con las preferencias de las personas target).

Los nuevos perfiles profesionales deberían tender a poner el acento sobre las responsabilidades siguientes de los operadores sociales: a) realizar una apropiada evaluación de las necesidades personales en colaboración con médicos, enfermeras y otros operadores, antes de decidir qué servicios deberían darse a la persona; b) diseñar paquetes de servicios acercando contemporáneamente las exigencias, tanto de las personas en estado de necesidad, como de aquéllas 
que les ayudan, es decir, paquetes desde una óptica de redes; c) asegurar la efectiva distribución de los servicios, no simplemente actuando como distribuidores directos, sino también desarrollando el rol de compradores, coordinadores y controladores de agencias de diverso tipo, de tal forma que puedan conseguir que los grupos primarios y secundarios sean lo más autónomos posible; d) hacer el seguimiento de la calidad y de la eficiencia de los servicios, siempre considerados desde una óptica de redes, y e) organizar incluso formas de intercambio social de care con los usuarios, y entre los usuarios, de tal manera que se creen recursos de redes.

Con toda probabilidad, las palabras clave en la formación de los operadores sociales en los próximos años serán: salvaguardar y elevar la calidad de los servicios; "normalizar» los cuidados, en el sentido de dar a todos las oportunidades para una vida «normal» en relación con las propias condiciones (en concreto, familiarizando los cuidados); invertir en un adiestramiento apropiado del personal; valorar las necesidades en relación con el «caso» singular y promover las capacidades de los operadores de formar paquetes de servicios personalizados a gestionar responsablemente en relación con el «caso» singular (difusión de las figuras del case coordinator y case manager); organizar servicioscentrados-en el-cliente, incluso iniciados-por-el-cliente.

\section{Bibliografía}

Ayala CaÑón, L.; Martínez LóPez, R. (1999). «La pobreza en España: evolución y factores explicativos». En: GARDE, J.A. Politicas sociales y Estado de bienestar en España. Informe 1999. Trotta.

Bulmer, M. (eds.) (1989). The Goals of Social Policy. Londres: Unwin Hyman.

CACHÓN, L. (1998). Nuevos yacimientos de empleo en España. Madrid: Ministerio de Trabajo y Asuntos Sociales.

CARRÀ, E. (2002). «Famiglie che si legano ad altre famiglie: il caso dell'associazionismo familiare in Lombardia». En: SCABINI, E.; ROSSI, G. La famiglia prosociale. Milán: Vita e Pensiero, p. 213-256.

CASTÓN, P.; RAMOS, M.; GARCÍA, J.M. (2004). «Mayores y discapacidad en el Estado de Bienestar». En: Herrera, M.; TRINIDAD, A. Administración Pública y Estado de Bienestar. Madrid: Thomson and Civitas.

De LeONARDIS, O. (1996). «Il welfare mix. Privatismo e sfera pubblica». Stato e Mercato, n. 46, abril, p. 51-75.

DenNis, I.; Catherine, A.N. (2003). «Poverty and social exclusion in the EU after Laeken». En: Statistics in focus. Population and Social Conditions, Theme 3-8/2003. Eurostat.

Di Nicola, P. (1998). Le reti come metafora dell'appartenenza. Analisi structturale e paradigma di rete. Milán: Angeli.

DONATI, P. (1986). Introduzione alla sociologia relazionale. Milán: Angeli.

- (1991). Teoria relaciónale della società. Milán: Angeli.

- (1993). La citadinanza societaria. Roma: Laterza.

- (2003). Manual de Sociología de la Familia. Pamplona: EUNSA.

ECSWTR (1987). Report of the Conference of European Ministers Responsible fon Social Affairs, Warsw, Poland, 6-10 de abril. 
FALCK, H. (1994). La prospettiva dell'appartenenza nel lavoro sociale. Milán: Vita e Pensiero.

FARGION, V. (1997). Geografia della cittadinanza sociale in Italia. Bolonia: Il Mulino. FLAQUER, L. (2004). «La articulación entre familia y el Estado de bienestar en los países de la Europa del sur». Papers, 73, p. 27-58.

Folgheraiter, F. (1994). Operatori sociali e lavoro di rete. Trento: Erickson.

Hernández Rodríguez, G. (2001). «Familia y Ancianos». Revista de Educación, n. 325, Madrid.

Herrera, M. (1998). «Nuevas dimensiones en políticas sociales: la community care». Revista Española de Investigaciones Sociológicas, n. 78, Madrid.

Herrera, M.; CASTÓN, P. (2003). Las politicas sociales en las sociedades complejas. Barcelona: Ariel.

Herrera, M.; Castón, P.; Ayuso, L.; PagéS, S. (2003b). «Familia y asistencia: una relación a revisar». Gestión y Análisis de Politicas Públicas, n. 23, p. 3-22.

Hirst, P. (1997). From statism to pluralism. Democracy, civil society and global politics. Londres: UCL Press.

- (1998). Social Welfare and Associative Democracy. Londres: Macmillan.

- (1999). "Associazionalismo: una strategia per la inclusion». Sociologia e politiche sociali, a. 2, n.3.

Hirst, P.; BADER, V. (2001). Associative Democracy, The Real Tirad Way. Londres: Fran Cass.

IgLesias de Ussel, J. (1998). Familia y cambio político en España. Madrid: Tecnos. IGLESIAS DE UsSel y otros (2001). La soledad de las personas mayores. Madrid: Ministerio de Trabajo y Asuntos Sociales.

Iglesias de Ussel, J.; MeIL, G. (2001). La politica familiar en España. Barcelona: Ariel.

LARAÑA, E.; GUSFIELD, J. (2001). Los nuevos movimientos sociales: De la ideología a la identidad. Madrid: CIS.

LAVILLE, J.L. (1992). Les services de proximité en Europe. París: Syros Alternatives.

Les perspectives d'avenir des divers systémes de sécurité sociale à la lumiére de l'evolution des structures familiales. Estrasburgo, 29 de marzo de 1988. Informe a cargo del profesor P. Gilliand, addendum II au CDSS-(88) 8.

López López, M.T. (2006). Políticas públicas y familia: Análisis de la situación en España. Madrid: Ediciones Cinca.

MeIL, G. (1999). La postmodernización de la familia española. Madrid: Acento.

- (2000). «Imágenes de la solidaridad familiar». Cuaderno de Opiniones y Actitudes, n. 30. Madrid: CIS.

- (2002). «Hogares nucleares y familias plurigeneracionales». En AA.VV. Estructura y cambio social. Libro homenaje a Salustiano del Campo. Madrid: CIS.

MeluCCI, A. (1989). Nomads of the present: Social movements and individual deeds in contemporary society. Londres: Century Hutchinson.

PAYNe, M. (1995). Social Work and Community Care. Londres: Macmillan.

- (1999). «I piani assistenziali individualizzati nelle cure di comunita». Intervención en el Congreso Internacional Gli operatori sociali nel welfare mix. Universidad de Trento, Facultad de Sociología, DU en Servicio Social, Riva del Garda, 20-21 de mayo.

Pérez Díaz, V.; Chuliá, E.; Álvarez Miranda, B. (1998). Familia y sistema de bienestar: La experiencia española con el paro, las pensiones, la sanidad y la educación. Madrid: Argentaria-Visor. 
Pinazo, S.; SÁnChez, M. (dir.) (2005). Gerontología. Actualización, innovación y propuestas. Madrid: Pearson-Prentice.

PRANDINI, R. (2006). «Framing Europe: L'emergere di un welfare state attivo "mother friendly" e le sue conseguenze per la famiglia». Sociología e Politiche Sociali, vol. 9-1, p. 69-108.

RAINERI, M.L. (2001). «Lavoro d'équipe e lavoro di rete nel welfare mix: different stili di interazione e di coordinamento". Sociologia e Politiche Sociali, 4, 3, p. 75-95. Rapport du Comité restreint d'experts sur la structure des ménages. Rapporteur W. Linke. Estrasburgo, 9 de febrero de 1989, CDPO (89) 2.

Rapport sur la politique de la famille. Raporteur M. Pini. Assemblée parlamentaire, $5 \mathrm{de}$ abril de 1988. Doc. 5870/1988.

Rapport sur les politiques familiales en Europe. Presentado por M. Michel Laroque. Estrasburgo, 20 de julio de 1989, CDPS (89) 7.

REQUENA, F. (1994). Amigos y redes sociales: Elementos para una sociología de la amistad. Madrid: Centro de Investigaciones Sociológicas.

REQUENA, M. (1999). «Pautas contemporáneas de evolución de los hogares en España». Revista Internacional de Sociología, n. 22.

Rossi, G. (1999). «Dall'assistenzialismo ai servizi alla persona». Sociologia e Politiche Sociali, 2, 1, p. 5-25.

SAlinas Ramos, F. (coord.) (2001). La evolución del Tercer Sector hacia la Empresa Social. Madrid: Plataforma para la promoción del voluntariado en España.

SANiCOla, L. (1990). Communitá e servici alla persona. Padua: CEDAM.

- (1993). L'intervento di rete. Nápoles: Liguori.

TRINIDAD REQUENA, A. (2005). La realidad económica y social de las personas mayores: Los jubilados tradicionales y los nuevos jubilados. Madrid: Instituto de Estudios Económicos.

Willmott, P. (1986). Social networks Informal Care and Plublic Policy. Londres: Policy Studies Institute. 